Proc. Int'l Conf. on Intelligent Robots and Systems (IROS '01), Maui, Hawaii, Oct. 29-Nov. 3, 2001.

\title{
A Precision Manipulator Module for Assembly in a Minifactory Environment
}

\author{
H. Benjamin Brown, Patrick M. Muir, Alred A. Rizzi, Maria C. Sensi, and Ralph L. Hollis \\ The Robotics Institute \\ Carnegie Mellon University
}

\begin{abstract}
In this paper we describe mechanical and electronic design of a precision, 2-DOF robot manipulator. The manipulator is one of a wide variety of modular robotic agents in the minifactory, a rapidly depoyable precision assembly system under development in our laboratory. The manipulator, in cooperation with another type of 2-DOF robot, termed a courier, can perform 4-DOF assembly operations emulating a SCARA. This arrangement provides increased precision, higher throughput, smaller footprint, and increased flexibility relative to the SCARA.
\end{abstract}

\section{Introduction}

Conventional 4-DOF precision assembly typically relies on SCARA robots [1] having precisions in the $50 \mu \mathrm{m}$ to $100 \mu \mathrm{m}$ range. Achieving higher precisions with the SCARA design is problematic, because of the proximal locations of the shoulder and elbow joints. The $x, y$ positioning resolution of the end effector depends critically on the resolutions of the shoulder and elbow joints (see Fig. 1).

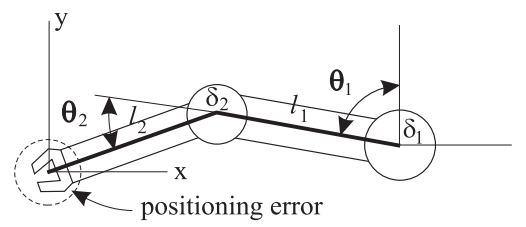

Figure 1: Geometry of SCARA robot configuration.

If the shoulder joint resolution is $\delta_{1}$ and the elbow joint resolution is $\delta_{2}$, then the worst case positioning uncertainty when the arm is extended is $\delta \approx\left(l_{1}+l_{2}\right) \sin \delta_{1}+l_{2} \sin \delta_{2}$. Additionally, several other important effects limit the SCARA's precision. These include some unavoidable static friction and "lost motion" or backlash in the shoulder and elbow transmissions which limit static performance, and transmission flexibility which limits dynamic performance. For assembly operations requiring greater precision than the SCARA can offer, it has been traditional to introduce separate precision $x, y$ motion stages with fixtures that are loaded and unloaded by the versatile SCARA. While workable, it would seem

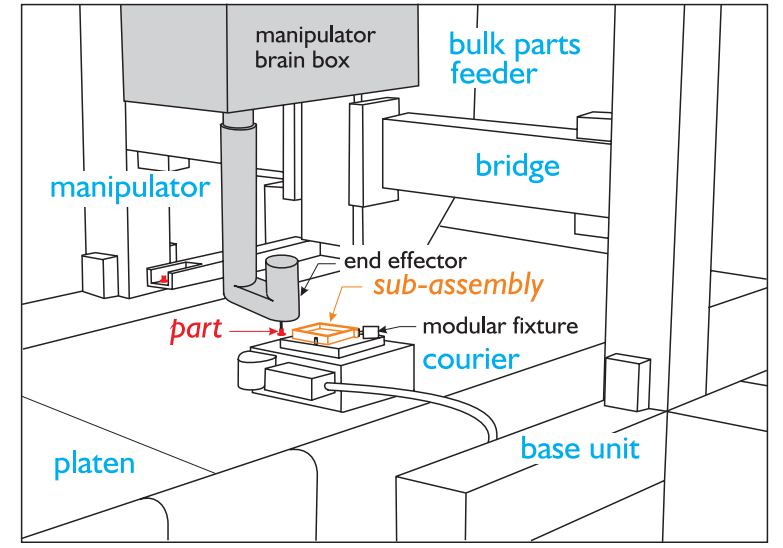

Figure 2: Small section of minifactory: A $z, \theta m a-$ nipulator and an $x, y$ planar robot courier perform a cooperative 4-DOF assembly operation. The manipulator, shaded in gray, is the subject of this paper.

advantageous to avoid this duplication of resources.

We have proposed a solution to the SCARA precision problem which involves pairs of 2-DOF robots working cooperatively to perform 4-DOF assembly operations [2, 3]. Figure 2 illustrates a small section of the tabletop minifactory assembly system under development in our laboratory [4, 5].* Product sub-assemblies are moved about on the factory floor, or platen, on the backs of courier robots based on closed-loop planar motors [6]. Manipulators which move vertically in $z$, and rotate in $\theta$ are clamped at fixed positions on modular bridges spanning the platen surfaces. The manipulators pick parts from local feeders and place them on the sub-assemblies. The combination of manipulator and courier is capable of precisions of the order of 100 times better than the SCARA, since unlike the SCARA all actuators and position/angle sensors in this arrangement are co-located. Unlike other 4-DOF robots based on planar motors, in our arrangement tasks can be pipelined between the two robots. Thus, the courier moves directly to the assembly location while at the same time the manipulator picks up the part from its local feeder, placing it as soon as the courier arrives [3].

In order to perform assembly at the micron level,

\footnotetext{
*Microdynamic Systems Laboratory http://ннн.cs.cmu.edu/ msl.
} 


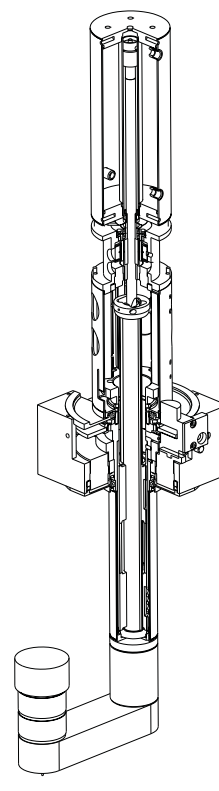

(a)

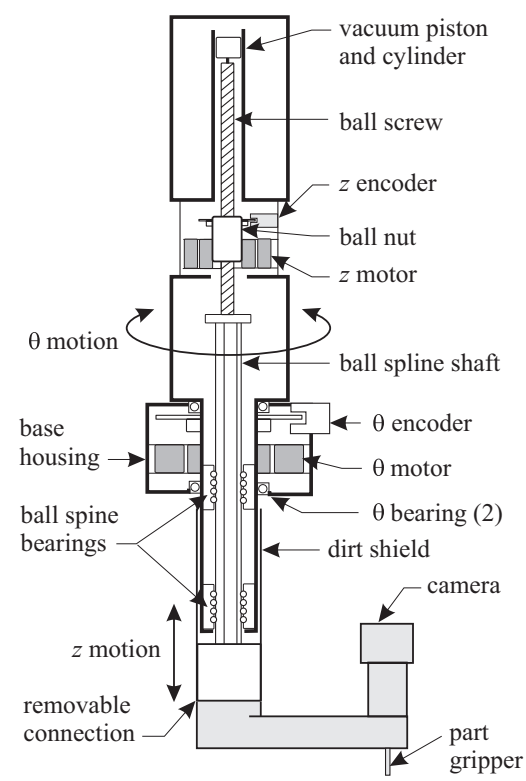

(b)
Figure 3: Manipulator design: (a) cutaway engineering model, $(b)$ schematic diagram; the end effector (shaded) is removable.

it is necessary for both the courier and manipulator robots to be carefully designed. We have previously developed couriers capable of $200 \mathrm{~nm}(1 \sigma)$ motion resolution and repeatability [6]. The remainder of this paper describes the design, construction, and operation of the manipulator half of this pair.

\section{Mechanical Design}

The manipulator, as shown in Fig. 3, has a rotational $(\theta)$ DOF greater than $360^{\circ}$ and a vertical $(z)$ translation of about $150 \mathrm{~mm}$. A typical end effector has a camera, illuminator and force sensor mounted on the end of a $100-\mathrm{mm}$ arm to facilitate parts picking. The manipulator and associated electronics are packaged in an enclosure or brainbox that can be handled easily by one person and mounted with an adjustable clamp to a minifactory bridge structure without tools. It is designed to be clean-room compatible.

A typical operating scenario is as follows. The end effector swings and moves downward to retrieve a part presented by a local parts feeder. The gripper moves down to a specified height, or until the integral force sensor detects the part. Vacuum is turned on to lift and hold the part. The part is lifted and the end effector swung to the nominal assembly location. Meanwhile, the courier positions a sub-assembly below, taking into account the measured, lateral position and orientation of the part with respect to the manipulator camera. As the manipulator carries the part downward for insertion, the courier moves to the correct lateral position using information from the manipulator. The component is pressed into position on the sub-assembly by the final $z$ motion, based on position and/or force signals. A puff of air releases the part, and the manipulator returns to the start position to begin the next cycle. The entire cycle takes less than a second.

Target specifications were selected to meet the stringent requirements for rapid, high-precision assembly. These included $0.4 \mathrm{~s}$ cycle time; lateral precision of $1 \mu \mathrm{m}$ at the end effector; vertical precision of $10 \mu \mathrm{m}$; minimal friction in both axes to facilitate position and force control; fail-safe mode to prevent drift on loss of power or vacuum; high stiffness; and minimal thermal drift. Further, the design needed to accommodate 5030 -gauge conductors plus six air/vacuum lines to the end effector. Unlike the $z, \theta$ axes of a SCARA, since the manipulator remains fixed in the minifactory, its overall height and weight can be arbitrarily large. The footprint, however, should be minimized to allow packing of many manipulators closely together. The target footprint was $150 \mathrm{~mm} \times 300 \mathrm{~mm}$ maximum. The weight was targeted at $15 \mathrm{Kg}$ maximum to allow mounting/unmounting by a single person.

Based on the requirements above, the critical design challenges included:

- Providing smooth, low friction $z$ translation and $\theta$ rotation as well as good lateral rigidity and repeatability at the end effector.

- Keeping masses, and especially the weight supported by the $z$ actuator, low to permit rapid cycling and minimize thermal dissipation in motors.

- Providing high resolution position sensing: $\sim 600,000$ counts per revolution in $\theta$ and $2-5 \mu \mathrm{m}$ in $z$.

- Keeping the manipulator and associated electronics compact and lightweight.

\subsection{Design Evolution}

To meet these design challenges, we undertook a development process that included consideration of numerous technologies, testing of concepts, modifying of available equipment for improved performance, and building two generations of prototypes. Concepts and technologies considered included cylindrical or planar air bearings for $z$ guidance; recirculating ball guides (linear ball bushings, rails, ball splines); lead screws, ball screws, cable and belt drives, and linear motors for $z$-actuation; cable, gear, belt and direct drives for $\theta$ actuation; and gravity compensation using springs, counterweights or air cylinders. 
Major problems were found or anticipated with most of the ideas considered. Air bearings, with excellent smoothness and precision, were rejected because of manufacturing difficulty, considering the required large $(0.25 \mathrm{~m})$ vertical movement. Lead screws were rejected because of excessive friction. Belt and chain drives were felt to have inadequate precision and stiffness.

A rough, first prototype was built using a railtype, recirculating-ball, linear guide system and cable drive for the $z$ motion. The rail, being relatively heavy, was fixed to ground, and a pair of ball-guide blocks mounted to a thin aluminum tube that surrounded both the guide rail and blocks, moved vertically. A small DC motor and wrapped-cable (figure8 ) drive at the lower end of the tube provided $\theta$ actuation of the end effector arm. The $z$ motion was smooth, but the lateral stiffness and repeatability at the tip were poor. Compliance in the $z$-drive cable corrupted servo performance, and the cable was vulnerable to wear and breakage. Overall, the design was cumbersome and difficult to package.

As a second approach, a very attractive idea was to use a recirculating-ball spline shaft as the vertically moving element, with the end effector attached to its bottom end. The shaft would be carried in a pair of ball nuts spaced apart for rigidity, mounted inside a rotary spindle. However, two major problems needed to be solved. First, the spline shaft needed a bore substantially larger than any commercially available for weight reduction and cable routing. Second, ball splines are known to experience binding, especially when used in a vertical orientation, precluding the smooth control required.

A ball spline (THK model LBS20) was selected for testing this approach. This was a $20 \mathrm{~mm}$ diameter unit having three projecting splines, six ball circuits, and a $12.7 \mathrm{~mm}(0.5 \mathrm{in}$.) bore. As received, the shafts were straight within $13 \mu \mathrm{m}(0.0005 \mathrm{in}$.) over $0.3 \mathrm{~m}$ (12 in.), but when bored they warped by as much as $750 \mu \mathrm{m}$ (0.030 in.), due apparently to residual stresses in the case-hardened steel. Numerous machining methods and straightening techniques were tried unsuccessfully to correct this problem. Finally a combination of moderate bending moment (to a nominal stress level of about $350 \mathrm{mP}$ or 50,000 PSI) and heating $\left(225^{\circ} \mathrm{C}\right.$ or $\left.350^{\circ} \mathrm{F}\right)$ was found to produce substantial changes in curvature, allowing return to near the original straightness. Apparently this combination tended to relax residual stresses by locally driving them beyond the yield point.

To address the binding problem, we considered ball-track smoothness and cleanliness; ball sphericity and size uniformity; frictional characteristics; and return-track smoothness. After many unsuccessful attempts, effort was focused on the inside of the spline nut. Small steps, found in the area where the balls en- tered the load and return tracks, were removed with a grinding wheel, and gentle entry/exit ramps were created and lapped with a brass rod, allowing each ball to assume/give up the load more gradually than in the original condition. With these modifications, friction was reduced to a consistent $.49 \mathrm{~N}$ or less, compared to original values of $2.4 \mathrm{~N}$ to $2.9 \mathrm{~N}$. ( Note that this treatment would adversely reduce the overturning rigidity in single-nut applications.)

\subsection{Design Details}

The second prototype manipulator was designed utilizing the modified ball spline as the basic element for $z$-translation, sliding in a pair of ball nuts, with a ball screw for actuation. Figure $3(a)$ is an engineering cutaway drawing showing the details of the mechanism, and Fig. 3(b) is a schematic of the internal operation. At its lower end, the tubular ball spline carries the end effector with $100 \mathrm{~mm}$ offset arm and camera. The ball nuts are mounted in a spindle supported on a pair of thin bearings, and actuated by a hollow, direct-drive motor. A vacuum-powered gravity compensator balances the weight of moving components to minimize the $z$-motor load and friction. The mechanism is mounted inside the brainbox housing motor amplifiers, control processors, interface electronics and air/vacuum components.

Rotary axis: The rotary axis comprises a direct drive motor, precision optical encoder, a pair of precision bearings and cushioned mechanical stops mounted in a square aluminum base housing. A direct-drive 12-pole pancake-style brushless DC torque motor (Hathaway Model HT03800) drives the $\theta$ axis, producing peak torque of $1.4 \mathrm{~N}-\mathrm{m}$ (200 oz.-in.) and maximum speed of 2800 RPM. The encoder, a Gurley Model 8710 with 9,000 optical lines, produces 720,000 counts per revolution with 20:1 interpolation of the sinusoidal output. This corresponds to a tangential resolution of $0.9 \mu \mathrm{m}$ at the gripper mounted on a $100 \mathrm{~mm}$ radius arm. The spindle carrying the motor, encoder and mechanical stop ring is mounted on a pair of $38.1 \mathrm{~mm}$ (1.5 in.) bore, thin-section, precision angular-contact bearings. The lower bearing mounts in a flexible diaphragm that has axial compliance to maintain bearing preload while providing the overturning stiffness needed at the end effector. A shuttle ring with rubber cushions allows $570^{\circ}$ of total rotation between stops.

Vertical axis: The vertical motion is guided by a modified recirculating ball spline (THK model LBS20) sliding in a pair of spline nuts mounted to the lower end of the rotary spindle. The spline shaft is bored to $12.7 \mathrm{~mm}(0.5 \mathrm{in}$.) diameter to minimize weight and provide clearance for wires and hoses routed to the end effector. A precision ball screw (Steinmyer Model 1214/4.8, $8 \mathrm{~mm}$ diameter, $4 \mathrm{~mm}$ 


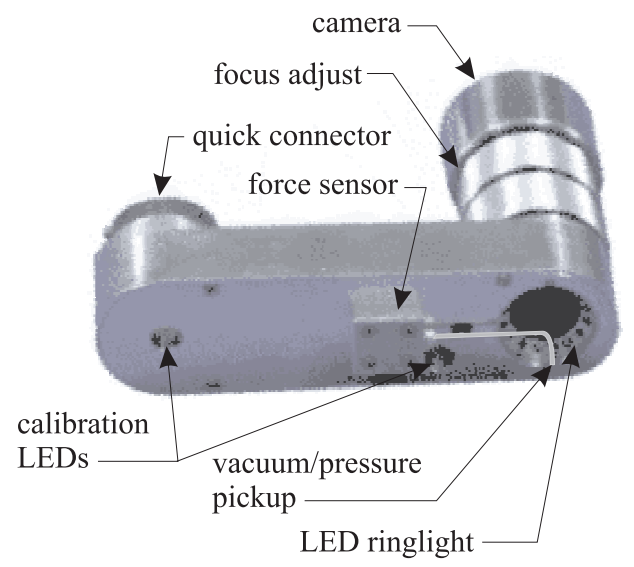

Figure 4: Photograph of an end effector.

lead) is pinned to the top end of the spline shaft and travels vertically with it. A small, hollow-rotor brushless DC motor (Hathaway Model HT01500), rotates the ball nut around the screw through a common, hollow spindle mounted on a pair of preloaded ball bearings. Because of the speed and acceleration requirements, axial forces can reach $230 \mathrm{~N}$ (50 lbs.) statically, or higher values dynamically, so all components must be ruggedly designed. An optical encoder (Hewlett Packard Model HEDS 5540) mounted above the ball nut produces 2,000 counts per revolution, giving $2 \mu \mathrm{m}$ vertical resolution. Also in the $z$-motor housing is a custom, vacuum-released brake that prevents drift of the $z$ axis on loss of electrical power or vacuum.

Gravity compensator: A vacuum-powered gravity compensator minimizes the required $z$-motor torque and frictional effects. The compensator utilizes an Airpot $^{\text {TM }}$ (Model S160, $16 \mathrm{~mm}$ bore) actuator comprising a precision glass cylinder and lowfriction graphite piston, with vacuum applied to the top of the piston. The glass cylinder is enclosed in and protected by the vacuum chamber, which provides a large volume to minimize pressure fluctuations as the piston moves up and down. The signal from a pressure sensor is used for controlling pressure through a three-way normally-closed solenoid valve. Maximum lift force is approximately $18 \mathrm{~N}$ at full vacuum. End-of-travel limit signals are obtained from two photoemitter/detector pairs sighting through the glass cylinder to determine the piston $z$-position.

End effector: A modular quick-release connector at the lower end of the manipulator carries the electrical power and signals, and air/vacuum to the modular end effector, shown in Fig. 4. A 3-ball kinematic mount and spring-loaded coupler assure repeatable attachment of the end effector. A typical end effector carries a small vacuum gripper tube mounted at a $100 \mathrm{~mm}$ radius from the manipulator centerline; a 3-axis force sensor integrated into the gripper; a

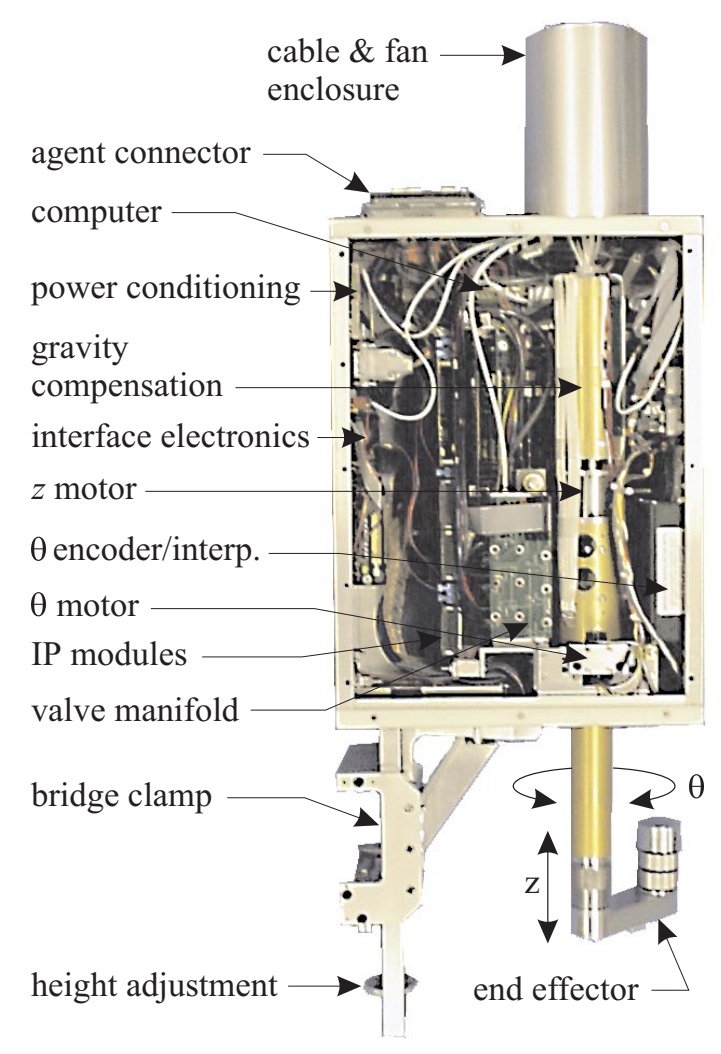

Figure 5: Photograph of the manipulator (cover removed).

camera and illuminator concentric with the gripper; a pair of LED "beacons" to allow optical relativeposition sensing by the courier below; and interface electronics.

Housing and clamp: A photograph of the complete manipulator is shown in Fig. 5. The brainbox encloses the manipulator mechanism, drive and sense electronics, computer, and all the equipment needed for independent operation and communication. The bridge clamp provides secure mounting of the manipulator to minifactory structural bridges, and vertical adjustment of $100 \mathrm{~mm}$ to accommodate different tasks (all without tools).

Cabling: One particularly tricky aspect of the design was accommodating the large number of connections needed for the end effector: 50 electrical conductors (10 five-conductor cables) and six air/vacuum hoses. From the end effector the wires and hoses pass through the bore of the spline shaft; through a rolling loop in the middle section that allows $z$ motion; to the outside of the tubular housing; through a hanging loop at the top of the gravity compensator that accommodates $\theta$ motion; and to their termination at the agent service connector. The cables/hoses are woven with thread into a flexible ribbon for the rolling loops. 


\section{Electronic Design}

To serve as a robotic agent within the minifactory environment, the manipulator is equipped with a workstation class computer augmented with interface and communications hardware. This single computer serves both as the control system for the electromechanical system described in Sec. 2 as well as its interface with peer robotic agents and factory-level functions. For this purpose, it has network interface devices and software which allow it to communicate with peers in order to describe itself (both geometrically and behaviorally), participate in simulation, and execute "programs" which describe how physical action should be performed and coordinated with that of its peers [7]. A pallet of intrinsic control policies for the manipulator include traditional PD and PID controllers, and a class of hybrid control policies [8] and task-oriented vision-based [9] and force-based [10] control policies. Sensing and actuation is done at a servo rate of $2 \mathrm{kHz}$.

Computational subsystem: All of our prototype minifactory agents are based on an ATX formfactor Motorola MTX-604-003 main-board with a single $300 \mathrm{MHz}$ Motorola 604e PowerPC. It includes 64 MB of on-board DRAM, three PCI expansion slots, an integrated 10/100 Mb Ethernet interface, a SCSI controller, two RS-232 interfaces and other standard features found in a workstation class computer.

The LynxOS ${ }^{\dagger}$ version 3.0.1 POSIX-conformant real-time operating system is used. This makes it possible for a single processor to execute real-time control tasks (executing at $\mathrm{kHz}$ rates) as well as overall system control and scripting, inter-device communication, and other high-level tasks. The choice to use a POSIX-conformant operating system has ensured portability of software onto the minifactory robotic agents, and has significantly reduced our software development and integration efforts.

Communications subsystem: Each agent in the minifactory environment must be able to coordinate and sequence its actions with those of its peers. This includes both abstract semantic scheduling (deciding what to do now) as well as higher-bandwidth sharing of sensing and actuation resources to accomplish coordinated motion, such as when a courier and manipulator cooperatively emulate a SCARA robot as described in Sec. 1. To accomplish this, each agent has two $100 \mathrm{Mb}$ Ethernet connections to the factory, as described in [11]. One is used for factory-wide latency-non-critical communication, and the other is used for local high-bandwidth low-latency communication between agents.

Interface subsystem: Beyond controlling the two brushless DC motors used to actuate the $z$ and $\theta$ axes of the robot, the manipulator has additional

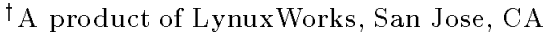

sensors and actuators suitable for performing tasklevel and diagnostic operations as shown in Fig. 5.

We use Industry-Pack (IP) modules on a carrier card for conversion between the analog and digital domain, encoder interfaces, and high-voltage digital outputs. The carrier card (Acromag model APC8620 ) is a PCI-based card which supports up to five IP modules. The manipulator robot uses four modules for all of its I/O. These include a general purpose counter/timer (Acromag IP480-6), 32 bits of highvoltage digital I/O (Acromag IP408), quadrature interfaces (SBS Technologies IP-QUADRATURE), and DAC/ADCs (SBS Technologies IP-PRECISION).

Control of the motor amplifiers is accomplished with two 12-bit DACs, along with 8 bits of digital I/O for amplifier safety control and monitoring. Each axis is equipped with a quadrature encoder whose output is routed to the quadrature interface IP module, which provides on-board 24-bit counters. Further, a number of ADC channels are used for self-test and monitoring to ensure functionality of the amplifier subsystems.

Actuation of the several pneumatic functions is performed with the high-voltage digital I/O IP module. With minimal additional protective circuitry this device is able to directly interface to the $12 \mathrm{~V}$ solenoids which operate the valves. These include six valves associated with applying high-pressure air or vacuum to three gripper functions, as well as operating the valve used in the gravity compensator. A pressure sensor is associated with each of these four functions and with both the high-pressure and vacuum supply manifolds. The readings from each pressure are acquired through the ADCs in the IPPRECISION module.

Finally, to support a variety of task-specific end effectors, additional digital I/O signals, analog inputs, and a video input are routed to the modular end effector connector. The video input is processed by a frame-rate direct-to-memory PCI-based video acquisition card (Imagenation PX610), and has been used to demonstrate high-precision image guided placement in the minifactory setting [9]. The analog inputs can be used to support a variety of task-specific sensors, including recently demonstrated force/moment sensing [10]. Finally, the digital input/outputs serve both to interface with an on-board flash memory device included in each end effector, as well as provide ancillary control functions for a variety of gripper capabilities.

\section{Results}

An important capability for the manipulator is that of holding a commanded $\theta$ while performing part placement. Figure 6 (top) shows estimated angular error as a function of manipulator angle with PID control 


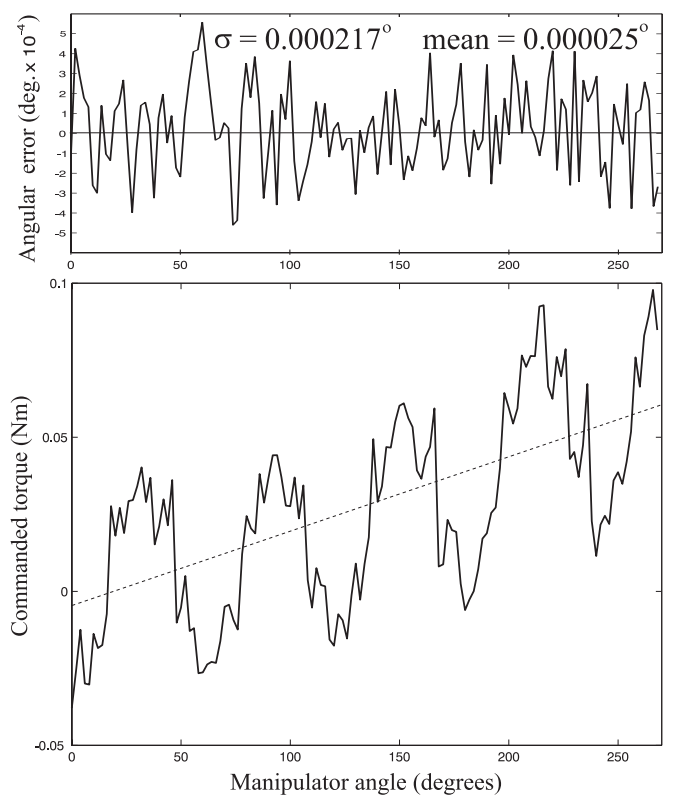

Figure 6: Manipulator $\theta$ axis positioning. Top plot: angular error; bottom plot: commanded torques to overcome motor detent ripple and cable windup.

with $K_{p}=250 \mathrm{Nm} / \mathrm{rad}, K_{d}=2.75 \mathrm{Nms} / \mathrm{rad}$, and $K_{i}=3000 \mathrm{Nm} / \mathrm{s}$. At $1 \sigma=0.000217^{\circ}$, the gripper on a $100 \mathrm{~mm}$ radius arm has $1 \sigma \approx 0.38 \mu \mathrm{m}$. Figure 6 (bottom) shows the torque command necessary to overcome a small motor torque ripple of period $60^{\circ}$, and a small linear spring force due to cable windup.

Another important capability is to make quick corrective motions prior to part placement. Figure 7 shows the step response under PD control with $K_{p}=70 \mathrm{Nm} / \mathrm{rad}$, and $K_{d}=.65 \mathrm{Nm} \mathrm{s} / \mathrm{rad}$, indicating a slew rate of about $1.2^{\circ} / \mathrm{s}$ for small moves.

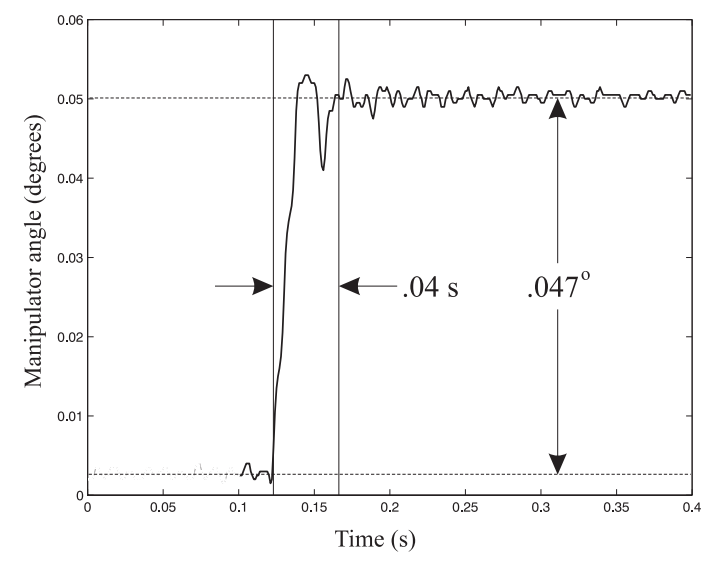

Figure 7: Manipulator $\theta$ small step response.

\section{Conclusions}

We have briefly presented design elements of a novel precision 2-DOF manipulator. There is much yet to be done, but preliminary performance results are encouraging and indicate that $\mu \mathrm{m}$-level operations can be performed. We have already demonstrated visionguided [9] and force-guided [10] operation. To date, we have built four copies of the manipulator described in this paper for use in the minifactory environment.

\section{Acknowledgements}

The authors gratefully acknowledge the contributions of Mike Chen, Mike Currie, Rich DeLuca, Jay Gowdy, Jeremy Horner, Chip Jansen, and Shinji Kume to the successful development of the manipulator. The project was supported in part by NSF grant DMI9527190.

\section{References}

[1] H. Makino and N. Furuya, "Selective compliance assembly robot arm," in Proc. First Int'l. Conf. on Assembly Automation, pp. 77-86, 1980 .

[2] R. L. Hollis and A. E. Quaid, "An architecture for agile assembly," in Proc. Am. Soc. of Precision Engineering. 10th Annual Mtg., (Austin, TX), October 15-19 1995.

[3] A. E. Quaid and R. L. Hollis, "Cooperative 2-DOF robots for precision assembly," in Proc. IEEE Int'l Conf. on Robotics and Automation, (Minneapolis), May 1996.

[4] A. A. Rizzi, J. Gowdy, and R. L. Hollis, "Agile assembly architecture: An agent-based approach to modular precision assembly systems," in IEEE Int'l. Conf. on Robotics and Automation, (Albuquerque), pp. 1511-1516, April 1997.

[5] R. L. Hollis and A. A. Rizzi, "Opportunities for increased intelligence and autonomy in robotic systems for manufacturing," in Eighth International Symposium of Robotics Research, (Hayama, Japan), October 3-7 1997.

[6] A. E. Quaid and R. L. Hollis, "3-DOF closed-loop control for planar linear motors," in IEEE Int'l Conf. on robotics and Automation, (Leuven, Belgium), pp. 2488-2493, May 1998.

[7] A. A. Rizzi, J. Gowdy, and R. L. Hollis, "Distributed programming and coordination for agent-based modular automation," in 9th Int'l. Symp. on Robotics Research, (Snowbird, Utah), October 1999.

[8] A. A. Rizzi, "Hybrid control as a method for robot motion programming," in IEEE Int'l Conf. on Robotics and Automation, (Leuven, Belgium), pp. 832-837, May 1998.

[9] M. L. Chen, S. Kume, A. A. Rizzi, and R. L. Hollis, "Visually guided coordination for distributed precision assembly," in IEEE Int'l. Conf. on Robotics and Automation, (San Francisco), pp. 1651-1656, April 2000.

[10] R. Deluca, A. A. Rizzi, and R. L. Hollis, "Forcebased interaction for distributed precision assembly," in Proc. Int'l Symp. on Experimental Robotics, (Honolulu, Hawaii), December 10-13 2000.

[11] S. Kume and A. A. Rizzi, "A high performance network infrastructure and protocols for distributed automation," in Proc. IEEE Int'l. Conf. on Robotics and Automation, 2001. (to appear). 